\title{
Neopterin is associated with hippocampal subfield volumes and cognition in HIV
}

Debra A. Fleischman, PhD, Konstantinos Arfanakis, PhD, Sue Leurgans, PhD, Sheila M. Keating, PhD, Melissa Lamar, PhD, David A. Bennett, MD, Oluwatoyin M. Adeyemi, MD, and Lisa L. Barnes, PhD

Neurol Neuroimmunol Neuroinflamm 2018;5:e467. doi:10.1212/NXI.0000000000000467
Correspondence

Dr. Fleischman

debra_fleischman@rush.edu

\section{Abstract}

\section{Objective}

HIV infection sets off an immediate immune response and inflammatory cascade that can lead to neuronal injury and cognitive impairment, but the relationship between immune markers, regional brain volumes, and cognition remains understudied in HIV-infected adults.

\section{Methods}

Cross-sectional associations were examined between serum immune markers of activation (neopterin) and inflammation (interleukin $[\mathrm{IL}]-1 \beta$, IL-6, tumor necrosis factor alpha, and C-reactive protein) with regional brain volumes (cortical, subcortical, total gray matter, hippocampus, and subfields) and cognition in 66 HIV-infected, virally suppressed, adults who underwent 3.0-T MRI as part of the Research Core of the Rush Center of Excellence on Disparities in HIV and Aging. Immune markers were assayed from frozen plasma, values were entered into linear regression models as predictors of regional brain volumes, and interactive effects of immune response and regional brain volumes on cognition were examined.

\section{Results}

No inflammatory marker was associated with any regional brain volume. Higher neopterin level was associated with lower total hippocampal, presubiculum, and cornu ammonis (CA) subfield volumes. Higher neopterin level and lower total hippocampal volume were independently associated with lower episodic memory, and neopterin level fully mediated the effect of hippocampal atrophy on episodic memory. Higher neopterin levels were associated with lower presubiculum, CA1, and CA4/dentate volumes and lower semantic memory, working memory, and global cognition.

\section{Conclusion}

Immune activation in response to HIV infection, measured by neopterin, has a deleterious and targeted effect on regional brain structure, which can be visualized with clinically available MRI measures of hippocampus and its subfields, and this effect is associated with lower cognitive function.

From the Rush Alzheimer's Disease Center (D.A.F., K.A., S.L., M.L., D.A.B., L.L.B.), Rush University Medical Center; the Department of Neurological Sciences (D.A.F., S.L., M.L., D.A.B., L.L.B.), the Department of Behavioral Sciences (D.A.F., M.L., L.L.B.), the Department of Preventive Medicine (S.L.), the Department of Diagnostic Radiology and Nuclear Medicine (K.A.), Rush University Medical Center; Ruth M. Rothstein CORE Center (O.M.A.); the Biomedical Engineering (K.A.), Illinois Institute of Technology, Chicago; the Blood Systems Research Institute (S.M.K.), San Francisco, CA; and the University of California at San Francisco (S.M.K.), Laboratory Medicine.

Funding information and disclosures are provided at the end of the article. Full disclosure form information provided by the authors is available with the full text of this article at Neurology.org/NN.

The Article Processing Charge was funded by the authors.

This is an open access article distributed under the terms of the Creative Commons Attribution-NonCommercial-NoDerivatives License 4.0 (CC BY-NC-ND), which permits downloading and sharing the work provided it is properly cited. The work cannot be changed in any way or used commercially without permission from the journal. 


\section{Glossary}

$\mathbf{C A}=$ cornu ammonis; CEDHA = Center of Excellence on Disparities in HIV and Aging; CRP = C-reactive protein; $\mathbf{C V}=$ coefficient of variation; FDR = false discovery rate; HAART = highly active antiretroviral therapy; IL = interleukin; INF = interferon; $\mathbf{T N F} \boldsymbol{\alpha}=$ tumor necrosis factor alpha.

It has long been known that HIV infection produces an almost immediate immune activation and inflammatory cascade that can persist in the face of effective highly active antiretroviral therapy (HAART) treatment ${ }^{1}$ and can lead to neuronal injury $^{2}$ and cognitive impairment. ${ }^{3}$ The earliest phase of the HIV immune response occurs when interferon-stimulated $(\mathrm{IFN}-\gamma)$ T-lymphocytes activate monocytes, macrophages, and dendritic cells to produce pteridines, a class of chemicals that stimulate the inflammatory cascade through the release of cytokines (e.g., interleukin [IL]-1 $\beta, \mathrm{IL}-6$, and tumor necrosis factor alpha $[\mathrm{TNF} \alpha]$ ) and acute phase reactants (e.g., C-reactive protein $[\mathrm{CRP}]$ ).

Neopterin, a pteridine produced in monocyte-derived macrophages ${ }^{4}$ and dendritic cells, ${ }^{5}$ is a well-established early marker of HIV-associated cellular immune activation. ${ }^{6}$ The neopterin level correlates with measures of viral load and CD4 count, is a sensitive measure of treatment efficacy and HIV disease course, ${ }^{7-9}$ and predicts HIV-related mortality. ${ }^{10}$ Neopterin level is associated with compromise of bloodbrain barrier integrity and generalized brain atrophy in $\mathrm{HIV}^{11}$ and HIV-associated cognitive impairment. ${ }^{2}$ Thus, neopterin level may signal activation of HIV-related immune mechanisms that can lead to neuronal injury, perhaps in regions most susceptible to metabolic disturbance, neurologic disease and alterations in cognitive function, but no study of the finer associations of the neopterin level with MRI-derived regional brain volumes and cognition in HIV has been reported.

Here, we examined relationships between immune activation measured by serum neopterin, inflammation measured by serum markers (IL-1 $\beta$, IL-6, TNF- $\alpha$, and CRP) known to be present in the HIV immune response, even with successful HAART treatment, and associated with brain abnormalities and cognitive impairment and decline, ${ }^{12-14} 3 \mathrm{~T}$ MRI-derived regional brain structure, and cognition in HIVinfected adults. Given the evidence that HIV is associated with increased levels of immune activation and chronic inflammation, we tested the hypothesis that higher level of immune activation, measured by neopterin, and higher inflammation, measured by these 4 inflammatory markers, would be specifically associated with volume loss in the hippocampus, a brain region known to be highly susceptible to metabolic stress and neurologic disease $\mathrm{e}^{15}$ and HIV infection. ${ }^{16}$ Furthermore, we hypothesized that this volume reduction would be associated with lower episodic memory, which depends critically on hippocampal integrity.

\section{Methods}

\section{Participants}

The study was conducted among participants in the Research Core of the Rush Center of Excellence on Disparities in HIV and Aging (CEDHA), a longitudinal study of older adults with HIV infection. HIV+ participants $(\mathrm{n}=175)$ were recruited from an urban infectious disease clinic. Eligibility requirements included documented HIV infection, African American, or white, age 50 and older, $\mathrm{CD} 4^{+} \geq 200$ cells $/ \mathrm{mm}^{3}$ on HAART or $\mathrm{CD}^{+} \geq 500$ HAART naive, and viral loads ranging from undetectable up to 50,000 copies. All participants underwent annual structured clinical evaluations that included an assessment of risk factors for cognitive decline, a battery of cognitive function tests, and a blood draw.

HIV+ participants who were willing to undergo MRI were approached if they were nondemented and able to give informed consent. Participants were excluded if there was any contraindication for MRI, history of head injury with loss of consciousness over 30 minutes, known large lesions, MS or hydrocephalus, seizure disorder, schizophrenia, current use of neuroleptic, narcotic medication, or anti-inflammatory medications. Seventy-eight of the 175 participants recruited into the research core were ineligible based on the above criteria. Of those 78 remaining, 11 participants were eligible but could not be contacted, 11 participants were reluctant to scan, 5 had unsuccessful scans, and 4 did not have blood available for assay, making the final sample 66 participants.

\section{Standard protocol approvals, registrations, and patient consents}

The study was approved by the Rush Institutional Review Board, and all participants signed study consent.

\section{Cognitive testing}

Each participant underwent cognitive function testing that included 18 tests of episodic memory, semantic memory, working memory, perceptual speed, and visuospatial ability. ${ }^{17}$ See supplementary materials for details, links.lww.com/ NXI/A47.

\section{Serum immune activation and inflammatory markers}

Neopterin was measured in undiluted plasma by ELISA (Alpco, Salem, NH; standard curve measuring $0.5-63 \mathrm{ng} / \mathrm{mL}$ with $3 \%$ intraplate and $7 \%$ interplate coefficient of variation $[\mathrm{CV}] \%)$. ELISA results were acquired using Molecular Devices E-Max plate reader with Softmax Pro v5.4. MILLIPLEX MAP Human High Sensitivity T Cell Panel was used to 
measure IL-1 $\beta$, IL- 6 , and TNF- $\alpha$ in undiluted plasma (Millipore, Billerica, MA; standard curve measuring $0.18-2,000 \mathrm{pg} /$ $\mathrm{mL}$ with $7 \%$ intra- and inter-plate CV). MILLIPLEX MAP Cardiovascular Disease Panel 3 was used to measure CRP in diluted plasma (1: 40,000) (standard curve measuring $0.01-50 \mathrm{ng} / \mathrm{mL}$ with $1 \%$ intraplate CV\% and $8 \%$ interplate CV\%). See supplementary materials for details, links.lww.com/NXI/A47.

\section{Brain image acquisition and processing}

All MRI data were collected on a 3-T Philips MRI scanner (Best, Netherlands) with a 32-channel head coil. See supplementary materials for further details (links.lww.com/NXI/A47) on MRI acquisition and postprocessing. ${ }^{18}$

\section{Statistical approach}

MRI data were transferred to SAS (SAS Institute, Inc., Cary, NC, 2012) for further data manipulation, linkage with demographic and serum immune marker data, and statistical analyses. Mean, SD, skewness coefficients, Q-Q plots, and histograms were obtained for age, education, CD4, serum markers, brain, and cognitive measures. Because the distributions of neopterin and CRP were markedly skewed, logarithmic transformations (base 10) were used in all analyses. Because the other 3 markers were below the detection limit in many participants, we used Spearman correlations in descriptive statistics, and we used dichotomized values (detected/not detected) in analytic models. First, we examined the correlations between demographic and disease characteristics with immune markers and with brain variables; then, we used regression models (with brain measures as outcomes) to examine the association of each of the immune markers with whole-brain measures (total cortical, total subcortical, total gray, and total hippocampus) with terms to correct for age, education, race, sex, and CD4 level. Based on the findings, we then examined the associations of neopterin with hippocampal subfield volumes, with false discovery rate (FDR) corrections for multiple comparisons. Finally, because of known associations of hippocampus volume with memory, we first considered episodic memory as the outcome of a multiple regression model with terms for neopterin and subfield volume and their interaction. In secondary models, we replaced episodic memory with semantic memory, working memory, perceptual speed, and visuospatial ability. All regression coefficients are reported per SD of the outcome; for continuous covariates, the regression coefficients are based on standardized covariates.

\section{Data availability}

Anonymized data not published within this article will be shared by request from any qualified investigator.

\section{Results}

Descriptive information for demographic, disease status, immune markers, brain volume, and cognitive measures is given in table 1 .
First, we examined correlations between the demographic, disease, and immune marker variables No immune marker or disease variable was associated with race or age ( $p s>0.05)$, and females had higher CRP level (female: mean $[\log 10] 4.17$, $\mathrm{SD}=0.55$; male: mean $=3.81, \mathrm{SD}=0.52, t=2.33, p=0.023$ ).

Persons with higher neopterin level had lower CD4 counts $(r=-0.369, p<0.01)$, but there was no association with nadir $\mathrm{CD} 4$ or disease duration. No inflammatory marker was associated with CD4 or any other disease variable. The inflammatory markers were significantly interrelated, but none was related to neopterin. Correlations between the immune marker variables are shown in supplementary materials, table e-1, links.lww.com/NXI/A47.

Next, we examined correlations between the demographic and disease severity variables with regional brain volumes. All selected regions had higher volumes with lower age and higher CD4 count $(p s<0.05)$. There were no significant associations with sex or race.

In a set of linear regression models, corrected for demographics (age, education, sex, and race) and CD4, we next examined associations between immune markers and total cortical, total subcortical, total gray, and total hippocampal volume. No immune marker was associated with total cortical, total subcortical, or total gray matter volume. Persons with higher neopterin levels had lower total hippocampal volumes (estimate $=0.398, \mathrm{SE}=0.128, p=0.003$ ). Estimates, SEs, and $p$ values are shown in table 2. The full model for neopterin and total hippocampal volume is shown in supplementary materials, table e-2, links.lww.com/NXI/A47. Because neopterin was the only immune marker associated with brain volume, no further analyses were performed with inflammatory markers. The remaining analyses concentrated on associations between neopterin, regional hippocampal volumes, and cognition.

Next, a finer examination of the associations of neopterin with hippocampal volume was undertaken using 7 subfield volumes: presubiculum, subiculum, cornu ammonis (CA) 1 , $\mathrm{CA} 2 / 3$, CA4/dentate, fimbria, and hippocampal fissure. Volumes of presubiculum, subiculum, and fimbria were higher with lower age $(p s<0.05)$. There were no associations with sex or race. Persons with higher CD4 count had higher volume in all fields except the hippocampal fissure $(p s<0.05)$.

In a series of linear regression models, corrected for demographic variables and $\mathrm{CD} 4$, persons with higher neopterin level had lower volumes of presubiculum, and the CA (CA1, $\mathrm{CA} 2 / 3$, and CA4/dentate) fields, after correcting probability levels for multiple comparisons using FDR. Estimates, SEs, and $p$ values for the regression models are shown in table 3 .

As a final step, we examined the associations of neopterin and regional brain volumes first with episodic memory in separate linear regression models controlled for demographic and 
Table 1 Demographic characteristics, HIV disease status, immune, brain, and cognitive measures $(n=66)$

\begin{tabular}{|c|c|c|c|c|}
\hline Measure & Mean & SD & Minimum & Maximum \\
\hline Age at scan & 58.5 & 5.3 & 50.6 & 71.3 \\
\hline Years education & 13.5 & 3.2 & 8.0 & 24.0 \\
\hline Male, $n$ (\%) & & & & \\
\hline Black, n (\%) & & & & \\
\hline CD4 & 638.5 & 313.5 & 228.0 & $1,872.0$ \\
\hline Nadir CD4 ${ }^{\mathrm{a}}$ & 276.4 & 191.2 & 6.0 & 756.0 \\
\hline Disease duration (y) & 16.0 & 7.1 & 1.0 & 32.0 \\
\hline Neopterin ${ }^{b}$ & 0.42 & 0.17 & 0.14 & 0.88 \\
\hline IL-1 $\beta, n$ (\%) & & & & \\
\hline IL-6, n (\%) & & & & \\
\hline TNF-a, n (\%) & & & & \\
\hline CRP $^{c}$ & 3.91 & 0.58 & 2.65 & 5.09 \\
\hline Cortical gray volume $^{d}$ & 262.99 & 21.58 & 211.03 & 308.28 \\
\hline Subcortical gray volume & 36.09 & 3.82 & 28.60 & 44.64 \\
\hline Total gray volume & 358.38 & 30.61 & 287.20 & 414.26 \\
\hline Hippocampal volume & 5.14 & 0.71 & 3.61 & 6.87 \\
\hline Presubiculum & 0.60 & 0.09 & 0.42 & 0.81 \\
\hline Subiculum & 0.80 & 0.11 & 0.58 & 1.10 \\
\hline CA1 & 0.42 & 0.05 & 0.29 & 0.53 \\
\hline $\mathrm{CA} 2 / 3$ & 1.74 & 0.17 & 0.87 & 1.63 \\
\hline CA4/dentate & 0.70 & 0.10 & 0.50 & 0.92 \\
\hline Fimbria & 0.07 & 0.03 & 0.01 & 0.12 \\
\hline Hippocampal fissure & 0.06 & 0.03 & 0.02 & 0.16 \\
\hline Global cognition $^{c}$ & 0.15 & 0.55 & -0.97 & 1.22 \\
\hline Episodic memory & 0.25 & 0.67 & -1.26 & 1.59 \\
\hline Semantic memory & 0.07 & 0.79 & -1.65 & 1.56 \\
\hline Working memory & 0.16 & 0.78 & -1.43 & 1.85 \\
\hline Perceptual speed & 0.09 & 0.82 & -1.68 & 1.85 \\
\hline Visuospatial ability & 0.04 & 0.80 & -1.38 & 1.57 \\
\hline
\end{tabular}

Abbreviations: $\mathrm{CA}=$ cornu ammonis; $\mathrm{CRP}=\mathrm{C}$-reactive protein; IL = interleukin; TNF- $\mathrm{a}=$ tumor necrosis factor alpha.

${ }^{a} \mathrm{n}=53$.

${ }^{\mathrm{b}}$ Log10-transform of units.

'All brain volumes are tenths of percents of intracranial volume.

${ }^{\mathrm{d}}$ All cognitive scores converted to Z-scores $($ mean $=0$ ).

disease variables, and then, in secondary analyses, with global cognition, semantic memory, working memory, perceptual speed, and visuospatial ability. Persons with lower neopterin levels (estimate $=-0.347, \mathrm{SE}=0.126, p<0.01$ ) and higher hippocampal volumes (estimate $=0.257, \mathrm{SE}=0.121, p<0.05$ ) had better episodic memory. When neopterin and hippocampal volumes were entered in a single model, the association between neopterin and episodic memory was slightly attenuated, but not eliminated (estimate $=-0.284$, SE $=$ $0.135, p=0.039$ ), but the association between hippocampal volume and episodic memory was eliminated $(p=0.22)$, suggesting that neopterin level fully mediated the association between hippocampal volume and episodic memory. The interaction of neopterin and hippocampal volume was not significant. Full models are shown in supplementary materials, table e-3, links.lww.com/NXI/A47. 
Table 2 Associations of immune markers and brain volumes (estimate, SE)

\begin{tabular}{lllll}
\hline & Cortical gray & Subcortical gray & Total gray & Hippocampus \\
\hline Neopterin & $-0.223(0.127)$ & $-0.080(0.131)$ & $-0.172(0.130)$ & $-0.398(0.128)^{\mathrm{b}}$ \\
\hline IL-1 $\beta$ & $0.049(0.236)$ & $0.230(0.236)$ & $0.093(0.239)$ & $0.072(0.252)$ \\
\hline IL-6 & $0.142(0.241)$ & $0.240(0.241)$ & $0.195(0.244)$ & $0.101(0.256)$ \\
\hline TNF-a & $-0.111(0.279)$ & $-0.049(0.281)$ & $-0.089(0.284)$ & $-0.156(0.297)$ \\
\hline CRP & $-0.073(0.116)$ & $0.051(0.117)$ & $-0.041(0.118)$ & $0.022(0.123)$ \\
\hline
\end{tabular}

Abbreviations: CRP = C-reactive protein; IL = interleukin; TNF- $\mathrm{a}=$ tumor necrosis factor alpha.

a Regression coefficients from separate models for brain volumes with a term for an immune marker, models controlled for age, sex, education, race, and CD4 status. IL1- $\beta$, IL-6, and TNF-a were included as binary variables (detected/not detected), and the coefficient is scaled to the SD of the brain volume. For neopterin and CRP, the coefficients are standardized regression coefficients.

${ }^{\mathrm{b}} \mathrm{p}<0.01$.

Interactions between the neopterin level and hippocampal subfield volumes were statistically significant for other cognitive domains. The standardized interaction term of the neopterin level with CA1 for semantic memory (estimate $=0.321, \mathrm{SE}=$ $0.114, p<0.01$ ), CA4/dentate for semantic memory (estimate $=0.29, \mathrm{SE}=0.14, p=0.04)$, presubiculum for global cognition (estimate $=0.215, \mathrm{SE}=0.106, p=0.047$ ), and presubiculum for working memory (estimate $=0.262, \mathrm{SE}=0.121, p=0.034$ ), were all statistically significant. Figure 1 illustrates the association of hippocampal subfield volumes to cognition for the 2 extreme quintiles (10th and 90th) of the neopterin level. The observation that the slopes for low levels of neopterin are negative and the slopes for high levels of neopterin are positive reflects the fact that all the interaction terms are positive.

\section{Discussion}

In this study, $66 \mathrm{HIV}$-infected adults who had higher serum neopterin levels, a measure of cellular immune activation, had

Table 3 Associations of neopterin and hippocampal subfield volumes (estimate, SE) ${ }^{\text {a }}$

\begin{tabular}{lllc}
\hline Subfield & Estimate & SE & Corrected $\boldsymbol{p}$ value $^{\mathbf{b}}$ \\
\hline Presubiculum & -0.31 & 0.12 & $\mathbf{0 . 0 1 5}$ \\
\hline Subiculum & -0.30 & 0.13 & 0.061 \\
\hline CA1 & -0.46 & 0.14 & $\mathbf{0 . 0 0 2}$ \\
\hline CA2/3 & -0.46 & 0.14 & $\mathbf{0 . 0 0 2}$ \\
\hline CA4/dentate & -0.46 & 0.13 & $\mathbf{0 . 0 0 3}$ \\
\hline Fimbria & -0.19 & 0.11 & 0.135 \\
\hline Fissure & -0.18 & 1.28 & 1.089 \\
\hline
\end{tabular}

Abbreviation: $\mathrm{CA}=$ cornuammonis.

astimated from separate models of standardized hippocampal subfield outcome with a term for standardized neopterin, controlled for age, sex education, race, and CD4 status. Thus, all coefficients in this table are expressed as standardized regression coefficients.

${ }^{b}$ FDR (false discovery rate) corrected for multiple comparisons.

Bold values are statistically significant. lower total hippocampal volume and lower presubiculum and CA subfield volumes. Episodic memory was better for persons with lower neopterin levels and for persons with higher hippocampal volumes. In mediation analyses, neopterin level fully accounted for the effect of total hippocampal volume on episodic memory. In hippocampal subfield analyses, persons with higher neopterin levels had lower CA1, CA4/dentate, and presubiculum volumes and lower semantic memory, working memory, and global cognition.

Neopterin production is the complex result of direct stimulation from IFN- $\gamma$, the only cytokine that directly induces it, ${ }^{19}$ and numerous indirect effects and interactions between multiple cytokines and thus reflects the entirety of immune network response on monocytes, macrophages, and dendritic cells. ${ }^{20}$ Neopterin is often elevated before antibody seroconversion or the onset of any clinical symptoms ${ }^{21}$ and hence may be an earlier and more sensitive indicator of HIVassociated immune response than any single, or any set of, inflammatory markers. ${ }^{6,20}$ In this study, we measured 4 inflammatory markers that are known to be present in HIV infection and associated with brain abnormalities and cognitive impairment and decline, IL- $1 \beta$, IL- 6 , TNF- $\alpha$, and CRP, but none was associated with brain volume. Only neopterin, a marker for immune activation, was associated with CD4, a marker of disease severity, and with regional brain volumes, specifically the hippocampus and its subfields.

No brain region is as susceptible to noxious insult as the hippocampus. ${ }^{15}$ The pyramidal neurons of the CA subfields, particularly $\mathrm{CA}_{1}{ }^{22}$ are well known to be vulnerable to anoxia-ischemia, ${ }^{23}$ metabolic stress (e.g., hypoglycemia ${ }^{24}$ ), microvascular pathology, ${ }^{25}$ and neurodegenerative disease such as Alzheimer disease and vascular dementia. ${ }^{26} \mathrm{HIV}$ viral load is high in the hippocampus ${ }^{27}$ and its subfields, with pyramidal neurons in CA3 possibly being more vulnerable to the virus than $\mathrm{CA1},{ }^{16}$ and reduced hippocampal volumes have been demonstrated in HIV-infected adults. ${ }^{28}$ The results of this study suggest that hippocampal atrophy that occurs in HIV is associated with immune activation, as measured by neopterin. 
Figure Association of hippocampal subfield volumes to cognition by neopterin level, volumes, and cognition adjusted for age, education, race, sex, and CD4
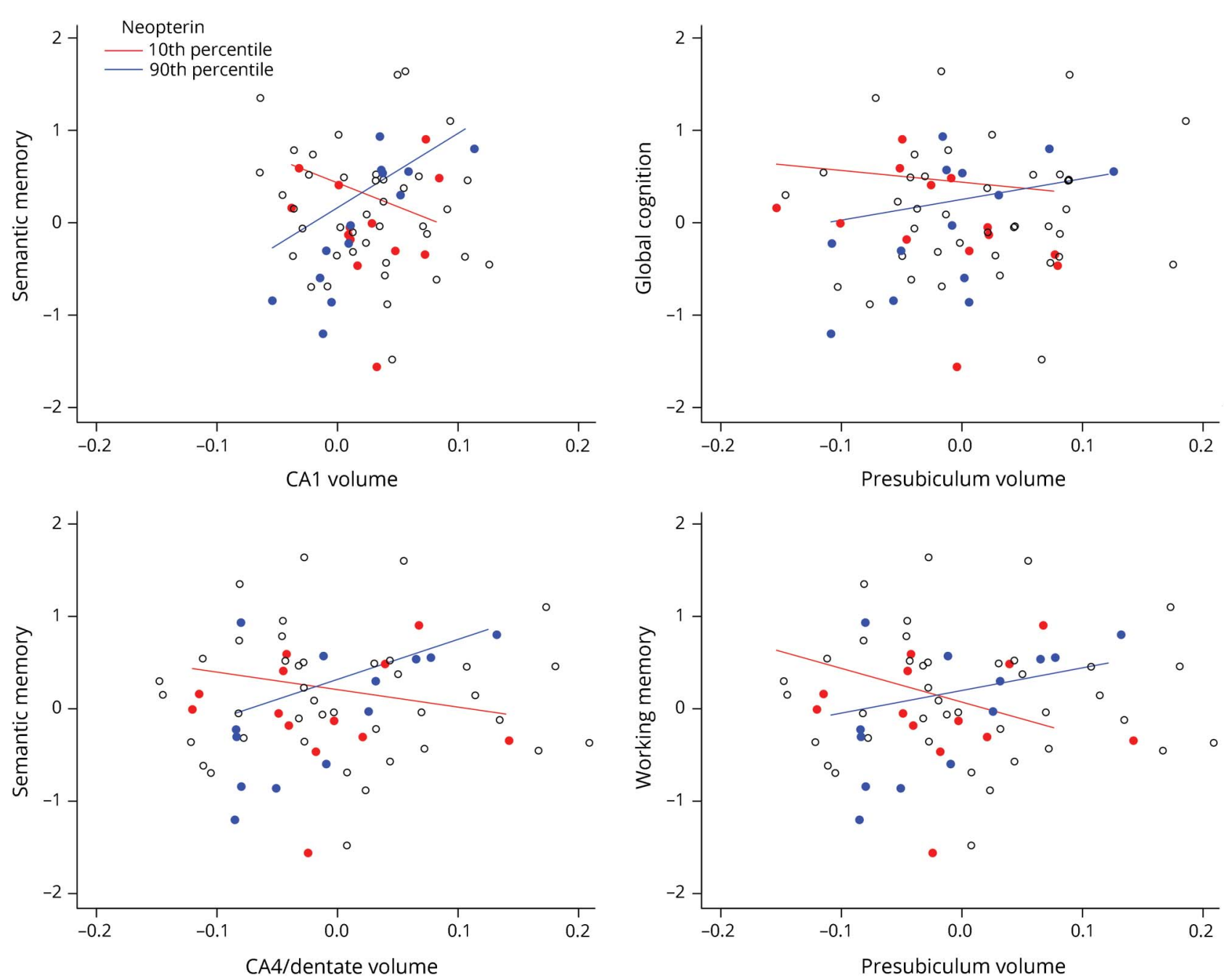

Colored lines show estimated cognition vs volume for extreme percentiles of neopterin (90th percentile in blue and 10th percentile in red). Solid points are data for persons in extreme quintiles (blue, 5th quintile; red, 1st quintile). Data for persons in the middle quintiles of neopterin are shown in open circles.

Hippocampal integrity is critical for normal memory function, $^{29}$ and in this study, hippocampal volume was, indeed, positively associated with episodic memory. Persons with higher neopterin levels had worse episodic memory, and, in mediation analyses, the neopterin level fully accounted for the effect of hippocampal atrophy on episodic memory. These results suggest that immune activation, as measured by neopterin, has a clear and robust deleterious effect on episodic memory in HIV-infected adults.

In hippocampal subfield analyses, persons with higher neopterin levels had lower volumes in 2 CA subfields (CA1 and $\mathrm{CA} / 4$ dentate) and lower semantic memory. Episodic and semantic memory are considered long-term, declarative memory processes known to be strongly dependent on hippocampal circuitry, ${ }^{30}$ particularly the CA subfields, with CA4/ dentate known to be a critical region for encoding information, and CA1 known to be a critical region for retrieval of that information. ${ }^{31}$ The results of this study suggest that immune activation in HIV-infected adults, measured by neopterin, may signal early volume reductions in hippocampal subfields that are responsible for normal encoding and retrieval of declarative information.

Although it has long been known that the hippocampus is critical for the encoding and retrieval of long-term declarative memories, more recently, the region has been shown to play a central role in other cognitive processes such as working memory $^{32}$ or the ability to maintain and manipulate multiple items simultaneously in short-term memory. The hippocampus is recruited in complex working memory span tasks that involve processing relations between item features, ${ }^{33}$ and the subiculum subfield has been shown to be preferentially activated in response to discriminating highly associated features from multiple viewpoints in a scene discrimination task. ${ }^{34}$ In this study, persons with higher levels of neopterin 
had lower volumes of the presubiculum, 1 of 4 component regions of the subiculum, and lower working memory and global cognition. This finding is consistent with the notion that immune activation, measured by neopterin, may flag HIV-associated neuronal injury in a vulnerable subfield and consequent risk for global cognition and working memory impairments. Overall, the results of this study suggest that neopterin level may serve as a proxy for ongoing risk of HIVassociated brain injury and neurocognitive disorder, but longitudinal studies are needed.

There are multiple mechanisms potentially linking neopterin biology with neuronal damage, neurodegeneration, and consequent cognitive impairment in HIV infection. One candidate mechanism is the disruption by chronic immune activation of the kynurenine pathway of tryptophan oxidative metabolism, which can induce cell damage through dysregulation of NMDA receptor function or generation of reactive oxygen species. ${ }^{35}$ Higher neopterin level is associated with lower serum tryptophan, brain atrophy, and dementia in HIV infection, ${ }^{11}$ and neurotoxic glutamate is known to be elevated in the CSF and plasma of patients with HIV. ${ }^{36} \mathrm{CAl}$ hippocampal cells have a high expression of NMDA receptors (which are critical for learning and memory) and are thus vulnerable to glutamate-mediated excitotoxicity. ${ }^{37}$ Indeed, the integrity of this pathway has been implicated in other medical conditions that involve chronic immune activation, brain injury, and cognitive impairment including Alzheimer disease, ${ }^{38}$ metabolic syndrome, and vascular cognitive impairment. ${ }^{39}$ Thus, it is feasible that chronic immune activation in HIV infection could be leading to neuronal loss and diminished cognitive function through an effect on the kynurenine pathway, but the biological mechanisms underlying the association between chronic immune activation, brain integrity, and cognitive function in HIV infection are complex and remain understudied.

This study had important strengths and limitations. First, the immune markers for this study were selected to target immune activation and inflammation, but only neopterin was associated with regional brain neuronal injury and cognitive impairment. Considering that the study participants were fully virally suppressed, low levels of plasma inflammatory markers may not have the dynamic range to identify associations with markers of HIV-induced pathology. Low concentrations for these cytokines are not uncommon in well-controlled HIV infection, and it is known that there is variable detectability of IL-6, TNF- $\alpha$, and IL- $1 \beta$ in HIV-infected individuals. The robust associations of neopterin with regional brain volumes and cognition shown here support previous studies demonstrating that subclinical CNS damage and consequent cognitive impairment do occur in HIV even when the virus is well controlled. ${ }^{2}$ Second, the number of subjects was small, and the lack of associations between regional brain volumes and the chosen inflammatory markers may be due to inadequate power, especially for the effects of the interaction of neopterin with brain volumes on cognition. However, the inflammatory markers were highly intercorrelated, and not related to neopterin, and associations with neopterin were robust, suggesting that the neopterin level may, indeed, be a window on an upstream immune response that is potentially damaging to the integrity of brain volume and cognition. Third, we used serum neopterin rather than CSF neopterin; however, a number of studies have shown that serum and CSF neopterin are correlated ${ }^{21}$ and that serum neopterin parallels HIV RNA levels. ${ }^{40}$ Fourth, the age range of our sample was limited, and the findings cannot be generalized to much younger or older HIV-infected adults. Fifth, the analyses did not adjust for premorbid general intelligence, which could influence individual level of cognitive reserve available to participants after contracting HIV. Sixth, the study is cross-sectional and therefore cannot demonstrate a causative relationship between neopterin level and brain volume and cognition. Finally, although our sample is small and cross-sectional, it was exceptionally well characterized within the parent CEDHA study, which is a longitudinal prospective study of HIV, and we will be able to perform longitudinal analyses in the future. Limitations notwithstanding, the results of this study suggest that the serum neopterin level and 3T neuroimaging, both of which are clinically available and relatively easy to obtain, may be useful in identifying those HIV-infected adults who are at risk of developing further neuronal injury and cognitive impairment. This information could prove invaluable for early implementation of targeted pharmacologic and behavioral interventions.

\section{Author contributions}

Study concept and design: D.A. Fleischman and K. Arfanakis. Analysis or interpretation of the data: D.A. Fleischman, K. Arfanakis, S. Leurgans, S.M. Keating, M. Lamar, D.A. Bennett, and L.L. Barnes. Drafting of the manuscript: D.A. Fleischman, K. Arfanakis, S. Leurgans, S.M. Keating, M. Lamar, D.A. Bennett, and L.L. Barnes. Statistical analysis: S. Leurgans. Obtained funding: D.A. Fleischman, K. Arfanakis, D.A. Bennett, O.M. Adeyemi, and L.L. Barnes.

\section{Acknowledgment}

The authors thank the staff of the Rush Center of Excellence on Disparities in HIV and Aging, the research staff of the Ruth M. Rothstein Core Center, and the staff of the Rush Alzheimer's Center, particularly Woojeong Bang, MS, for statistical analysis and Niranjini Rajendran, MS, for image postprocessing.

\section{Study funding}

This study was supported by the National Institute of Minority Health and Health Disparities grant P20MD6886; National Institute of Aging grant P30AG010161; and Illinois Department of Public Health.

\section{Disclosure}

D.A. Fleischman received research support from the National Institute of Minority Health and Health Disparities. K. Arfanakis served on the editorial board of Brain Imaging and Behavior and 
received research support from the NIA and NINDS. S. Leurgans serves as an associate editor of statistics for Neurology and received research support from the NIH. S.M. Keating received research support from the NIH/NHLBI, NIH/NIAID, US FDA, amfAR Institute for HIV Cure. M. Lamar received research support from the NIA, NINDS, and NHLBI. D.A. Bennett served on the scientific advisory board of Vigorous Minds, Takeda Pharmaceuticals, AbbVie, Indian Institute of Science, MCSA, Columbia ADRC, CCNA, CIMA-Q WFU, Emory, MODEL-AD, REGARDS, HRS, MIDUS, and National Advisory Council on Aging; serves on the editorial board of Neurology, Current Alzheimer Research, and Neuroepidemiology; and received research support from the NIH. O.M. Adeyemi consulted for Gilead and received research support from AbbVie. L.L. Barnes serves on the editorial board of the Journal of Aging and Health and received research support from the NIA. Full disclosure form information provided by the authors is available with the full text of this article at Neurology.org/NN.

Received October 16, 2017. Accepted in final form April 4, 2018.

\section{References}

1. Heaton RK, Clifford DB, Franklin DR Jr, et al; CHARTER Group. HIV-associated neurocognitive disorders persist in the era of potent antiretroviral therapy: CHARTER Study. Neurology 2010;75:2087-2096.

2. Anesten B, Yilmaz A, Hagberg L, et al. Blood-brain barrier integrity, intrathecal immunoactivation, and neuronal injury in HIV. Neurol Neuroimmunol Neuroinflamm 2016;3:e300.

3. Ances BM, Ellis RJ. Dementia and neurocognitive disorders due to HIV-1 infection.Semin Neurol 2007;27:86-92.

4. Huber C, Batchelor JR, Fuchs D, et al. Immune response-associated production of neopterin:release from macrophages primary under control of interferon-gamma. J Exp Med 1984;160:310-316.

5. Wirleitner B, Reider D, Ebner S, et al. Monocyte-derived dendritic cells release neopterin. J Leukoc Biol 2002;72:1148-1153.

6. Wachter H, Fuchs D, Hausen A, Reibnegger G, Werner ER. Neopterin as a marker for activation of cellular immunity: immunologic basis and clinical application. In: Spiegel HE, editor. Advances in Clinical Chemistry, vol 27. San Diego, CA: Academic Press; 1989:82-141.

7. Fuchs D, Spira TJ, Hausen A, et al. Neopterin as a predictive marker for disease progression in human immunodeficiency virus type 1 infection. Clin Chem 1989;35:1746-1749.

8. Mildvan D, Spritzler J, Grossberg SE, et al. Serum neopterin, an immune activation marker, independently predicts disease progression in advanced HIV-1 infection. Clin Infect Dis 2005;40:853-858.

9. Wirleitner B, Schroecksnadel I, Winkler C, Fuchs D. Neopterin in HIV-1 infection. Mol Immunol 2005;42:183-194.

10. Stein DS, Lyles RH, Graham NM, et al. Predicting clinical progression or death in subjects with early-stage human immunodeficiency virus (HIV) infection: a comparative analysis of quantification of HIV RNA, soluble turmor necrosis factor type II receptors, neopterin, and B2-microglobulin. Multicenter AIDS Cohort Study. J Inf Dis 1997; 176:1161-1167.

11. Fuchs D, Moller AA, Reibnegger G, Stockie E, Werner ER, Wachter H. Decreased serum tryptophan in patients with HIV-1 infection correlates with increased serum neopterin and with neurologic/psychiatric symptoms. J Acquir Immune Defic Syndr 1990;3:873-876

12. Bridges SL, Jenq G, Moran M, Kuffner T, Whitworth WC, McNicholl J. Singlenucleotide polymorphisms in tumor necrosis factor receptor genes: definition of novel haplotypes and racial/ethnic differences. Arthritis Rheum 2002;46:2045-2050.

13. Khera A, McGuire DK, Murphy SA, et al. Race and gender differences in C-reactive protein levels. J Am Coll Cardiol 2005;46:464-469.
14. Cox DE, Hoffman SC, DiMercurio BS, et al. Cytokine polymorphic analyses indicate ethnic differences in the allelic distribution of interleukin-2 and interleukin-6. Transplantation 2001;72:720-726.

15. Bartsch T. The hippocampus in neurological disease. In: Bartsch T, editor. The Clinical Neurobiology of the Hippocampus: An Integrative View. Oxford, United Kingdom: Oxford University Press; 2012:200-205.

16. Torres-Munoz J, Stockton P, Tacoronte N, Roberts B, Maronpot RR, Petito CK. Detection of HIV-1 gene sequences in hippocampal neurons isolated from postmortem AIDS brains by laser capture microdissection. J Neuropathol Exp Neurol 2001;60:885-892.

17. Krueger KR, Adeyemi O, Leurgans S, et al. Association of cognitive activity and neurocognitive function in blacks and whites with HIV. AIDS 2017;31:437-441.

18. Malone IB, Leung KK, Clegg S, et al. Accurate automatic estimation of total intracranial volume: a nuisance variable with less nuisance. Neuroimage 2015;104:366-372.

19. Werner-Felmayer G, Werner ER, Fuchs D. Hausen A, Reibnegger G, Wachter H. Neopterin formation and tryptophan degradation by a human myelomonocytic cell line (THP-1). Cancer Res 1990;50:2863-2867.

20. Sucher R, Schroecksnadel K, Weiss G, Margreiter R, Fuchs D, Brandacher G. Neopterin, a prognostic marker in human malignancies. Cancer Lett 2010;287:13-22.

21. Sonnerborg AB, von Stedingk LV, Hansson LO, Strannegard OO. Elevated neopterin and beta 2-microglobulin levels in blood and cerebrospinal fluid occur early in HVIinfection. AIDS 1989;3:277-283.

22. Nikonenko AG, Radenovic L, Andjus PR, Skibo GG. Structural features of ischemic damage in the hippocampus. Anat Rec (Hoboken) 2009;292:1914-1921.

23. Schmidt-Kastner R, Freund TF. Selective vulnerability of the hippocampus in brain ischemia. Neuroscience 1991;40:599-636.

24. Auer RN, Wieloch T, Olsson Y, Siesjo BK. The distribution of hypoglycemic brain damage. Acta Neuropathol 1984;64:177-191.

25. Kril J, Patel S, Harding A, Halliday G. Patients with vascular dementia due to microvascular pathology have significant hippocampal neuronal loss. J Neurol Neurosurg Psychiatry 2002;72:747-751.

26. Zarow C, Vinters HV, Ellis WG, et al. Correlates of hippocampal neuron number in Alzheimer's disease and ischemic vascular dementia. Ann Neurol 2005;57:896-903.

27. Fujimura RK, Goodkin K, Petito CK, et al. HIV-1 proviral DNA load across neuroanatomic regions of individuals with evidence for HIV-1 associated dementia. J Acquir Immune Defic Syndr Hum Retrovirol 1997;16:146-152.

28. Kallianpur KJ, Shikuma C, Kirk GR, et al. Peripheral blood HIV DNA is associated with atrophy of cerebellar and subcortical gray matter. Neurology 2013;80: 1792-1799.

29. Bartsch T, Schonfeld R, Muller FJ, et al. Focal lesions of human hippocampal CA1 neurons in transient global amnesia impair place memory. Science 2010;328:1412-1415.

30. Fleischman DA, Wilson RS, Gabrieli JD, Schneider JA, Bienias JL, Bennett DA. Implicit memory and Alzheimer's disease neuropathology. Brain 2005;128:2006-2015.

31. Eldridge LL, Engel SA, Zeineh MM, Bookheimer SY, Knowlton BJ. A dissociation of encoding and retrieval processes in the human hippocampus. J Neurosci 2005;25: 3280-3286.

32. Faraco CC, Linsworth N, Langley J, et al. Complex span tasks and hippocampal recruitment during working memory. Neuroimage 2011;55:773-787.

33. Wilson TW, Proskovec AL, Heinrichs-Graham E, et al. Aberrant neuronal dynamics during working memory operations in the aging HIV-infected brain. Sci Rep 2017; 7 41568 .

34. Hodgetts CJ, Voets NL, Thomas AG, Clare S, Lawrence AD, Graham KS. Ultra-highfield fMRI reveals a role for the subiculum in scene perceptual discrimination. J Neurosci 2017;37:3150-3159.

35. Stone TW, Darlington L. Pharmacology of the kynurenine pathway. Int Congress Ser 2007;1304:298-304.

36. Farrarese C, Aliprandi A, Tremolizzo L, et al. Increased glutatmate in CSF and plasma of patients with HIV dementia. Neurology 2001;57:671-675.

37. Wang X, Ranu P, Chen X-W, Limpeanchob N, Kuman KN, Michaelis EK. High intrinsic oxidative stress may underlie selective vulnerability of the hippocampal CAl region. Mol Brain Res 2005;140:120-126.

38. Pawlek GE, Bien B, Pawlek D. Kynurenine and its metabolites in Alzheimer's disease patients. Adv Med Sci 2010;55:204-211.

39. Oxenkrug GF. Interferon-gamma-inducible kynurenines/pteridines inflammation cascade: implication for aging and aging-associated psychiatric medical disorders. J Neural Transm 2011;118:75-85.

40. Hagberg L, Cinque P, Gisslen M, et al. Cerebrospinal fluid neopterin: an informative biomarker of central nervous system immune activation in HIV-1 infection. AIDS Res Ther 2010;7:15. 


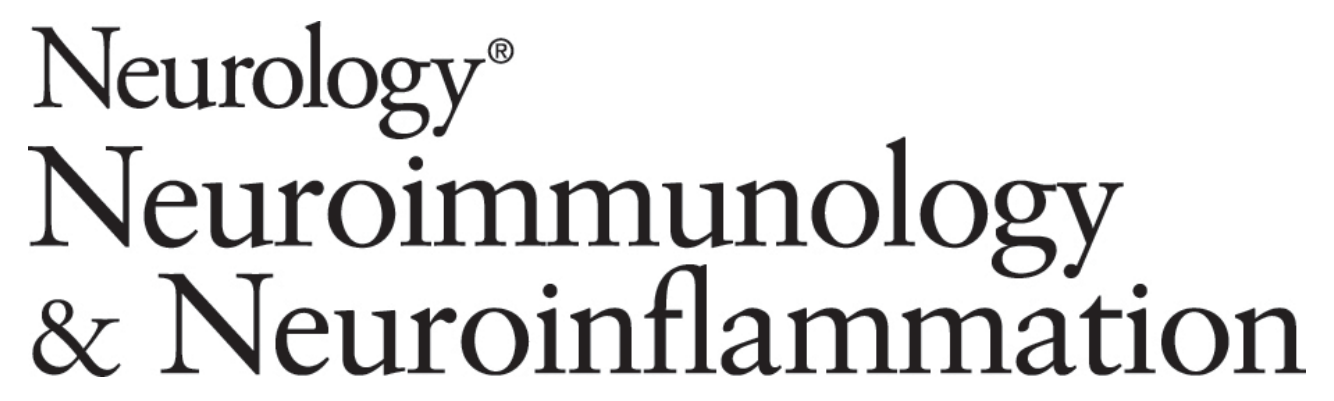

Neopterin is associated with hippocampal subfield volumes and cognition in HIV Debra A. Fleischman, Konstantinos Arfanakis, Sue Leurgans, et al.

Neurol Neuroimmunol Neuroinflamm 2018;5;

DOI 10.1212/NXI.0000000000000467

This information is current as of June 11, 2018

Neurol Neuroimmunol Neuroinflamm is an official journal of the American Academy of Neurology.

Published since April 2014, it is an open-access, online-only, continuous publication journal. Copyright

Copyright $\odot 2018$ The Author(s). Published by Wolters Kluwer Health, Inc. on behalf of the American

Academy of Neurology.. All rights reserved. Online ISSN: 2332-7812.

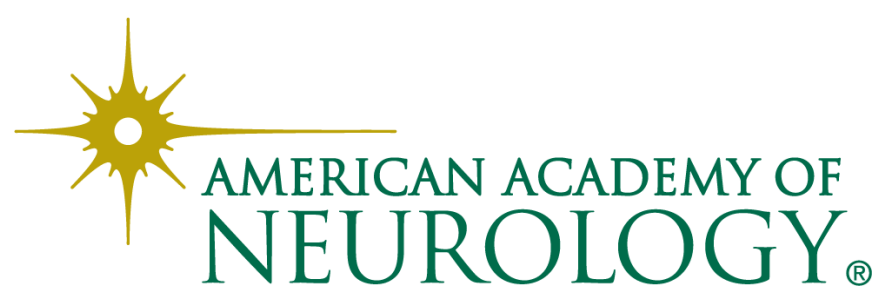




\section{Updated Information \& Services}

References

Subspecialty Collections

Permissions \& Licensing

Reprints including high resolution figures, can be found at: http://nn.neurology.org/content/5/4/e467.full.html

This article cites 39 articles, 8 of which you can access for free at: http://nn.neurology.org/content/5/4/e467.full.html\#\#ref-list-1

This article, along with others on similar topics, appears in the following collection(s):

All Clinical Neurology

http://nn.neurology.org//cgi/collection/all_clinical_neurology

All Cognitive Disorders/Dementia

http://nn.neurology.org//cgi/collection/all_cognitive_disorders_dementi a

All Immunology

http://nn.neurology.org//cgi/collection/all_immunology

\section{HIV}

http://nn.neurology.org//cgi/collection/hiv

Volumetric MRI

http://nn.neurology.org//cgi/collection/volumetric_mri

Information about reproducing this article in parts (figures,tables) or in its entirety can be found online at:

http://nn.neurology.org/misc/about.xhtml\#permissions

Information about ordering reprints can be found online:

http://nn.neurology.org/misc/addir.xhtml\#reprintsus

Neurol Neuroimmunol Neuroinflamm is an official journal of the American Academy of Neurology.

Published since April 2014, it is an open-access, online-only, continuous publication journal. Copyright

Copyright $\odot 2018$ The Author(s). Published by Wolters Kluwer Health, Inc. on behalf of the American

Academy of Neurology.. All rights reserved. Online ISSN: 2332-7812.

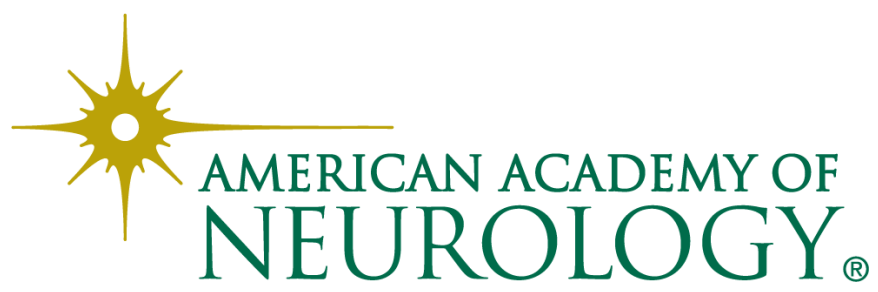

